\title{
Modeling and Design of a Spring-loaded, Cable-driven, Wearable Exoskeleton for the Upper Extremity
}

\author{
Lelai Zhou ${ }^{1}$ Shaoping Bai ${ }^{2}$ Michael Skipper Andersen ${ }^{2}$ John Rasmussen ${ }^{2}$ \\ ${ }^{1}$ Vestas Wind Systems, Denmark. E-mail: LAZHU@vestas.com \\ ${ }^{2}$ Department of Mechanical and Manufacturing Engineering, Aalborg University, 9220 Aalborg, Denmark. E-mail: \\ $\{s h b, m s a, j r\} @ m$-tech.aau.dk
}

\begin{abstract}
An approach to the design of wearable exoskeletons on the basis of simulation of the exoskeleton and a human body model is proposed in this paper. The new approach, addressing the problem of physical human-exoskeleton interactions, models and simulates the mechanics of both the exoskeleton and the human body, which allows designers to effectively analyze and evaluate an exoskeleton design for their function in concert with the human body. A simulation platform is developed by integrating a biomechanical model of the human body and the exoskeleton. With the proposed approach, an exoskeleton is designed for assisting patients with neuromuscular injuries. Results of the analysis and optimization are included.
\end{abstract}

Keywords: Exoskeleton; cable-driven; musculoskeletal model; optimization; biomechanics

\section{Introduction}

Exoskeleton robots have prospective applications in rehabilitation and patient assistance. They can allow users to retain independent living by regaining mobility and manipulability (Haumont et al., 2011).

A number of exoskeletons or robotic exoskeleton systems have been developed in recent years and can be categorized into two major groups. One group is passive exoskeletons. Wilmington Robotic Exoskeleton (WREX), a two-segment, 4-DOF (degree of freedom) passive orthosis provided by Nemours (Rahman and et al., 2006), is a modular, body-powered orthosis which can be mounted on a person's wheelchair or to a body jacket. WREX uses linear elastic elements to balance the effects of gravity in three dimensions. A variable impedance-powered elbow exoskeleton named NEUROExos (Lenzi et al., 2011) was developed for the rehabilitation task of stroke patients. The robot utilizes a double shell link structure and 4-DOF passive mechanism, which has good kinematic compatibility with human anatomy. NEUROExos makes use of an adaptive, passive-compliant actuator through a bioinspired antagonistic non-linear elastic actuation system. An upper limb exoskeleton with 3-DOF shoulder joint and 1-DOF elbow joint has been designed (Wu et al., 2011). The grounding device can increase resistance through adjustment of the spring length to train more muscle groups. Passive exoskeletons have the advantage that they do not rely on actuation devices to power the system. This allows for compact and lightweight design.

Another group is the active exoskeletons. The ARMin III (Nef et al., 2009) is an arm therapy exoskeleton robot with three actuated DOFs for the shoulder and one for the elbow. It was designed to improve the rehabilitation process in stroke patients. The 
IntelliArm (Ren et al., 2009) is a whole arm robot, which has a total of eight actuated DOFs and another two passive DOFs at the shoulder. Besides, the IntelliArm has an additional DOF for hand opening and closing. Several other types of actuated exoskeleton robots are also proposed, such as ABLE (Garrec et al., 2008), CADEN-7 (Perry et al., 2007), MGA (Carignan et al., 2009), RehabExos (Vertechy et al., 2009), and Pneu-WREX (Wolbrecht et al., 2008). The active exoskeletons usually have rigid mechanical joints driven by electric motors. A detailed review of the state-of-theart exoskeleton robots for the upper limb is available (Lo and Xie, 2012).

Compared with passive exoskeletons, the active exoskeletons can provide higher force augmentation. However, they require electric actuators and power supply, which make the system heavy and reduce the wearability. In the application of patient assistance in daily living, passive exoskeletons can be wearable and designed with simple and lightweight structures. The purpose of this work is to design a wearable passive exoskeleton.

Cable-driven systems have the advantage of lightweight distal force transfer and are compatible with the human body's anatomical structure. A 4DOF cable-driven exoskeleton for the upper arm was presented in (Agrawal et al., 2009), and recently upgraded to seven DOFs (Mao and Agrawal, 2012). This design makes use of cables driven by electric motors to actuate the exoskeleton and requires grounding support for the motors and power supply, which precludes walking freely while wearing the exoskeleton.

As the passive exoskeleton will work under an assistive mode, a better understanding of the biomechanics of the upper extremity motion and sensory mechanisms is critical to the exoskeleton design. Some relevant works can be found in the literature. Muscle forces of the human arm with an exoskeleton were experimentally tested (Gallagher et al., 2013), where individual muscle force was optimized through optimal motor-task of the exoskeleton. A musculoskeletal model was developed to model interactions between the human and rehabilitation devices (Lee et al., 2009; Bai and Rasmussen, 2011).

In this work, a cable-driven, wearable exoskeleton actuated by gravity-compensating spring forces is designed. A simulation platform is developed for the modeling of the physical human-exoskeleton interaction. An advanced biomechanical model of the upper extremity is developed by virtue of the AnyBody Modeling System (AMS) (AnyBody Technology A/S, Aalborg, Denmark), which provides a detailed understanding of the mechanics of the human-exoskeleton system, i.e., the exoskeleton and the human body. Determination of the design parameters of the exoskeleton is formulated and solved as an optimization problem.

\section{Design of the Exoskeleton}

A wearable upper extremity exoskeleton has to be kinematically compatible with the human arm. The human arm is constructed for dexterity with most of the muscles placed proximally with respect to the joints they actuate to minimize distal mass. Similarly, the fundamental design principle of the exoskeleton is to place springs storing elastic energy close to the body and transfer their effect via flexible cables to the distal parts of the exoskeleton. The exoskeleton has three cuffs, namely, the armor cuff, the elbow upper and lower brackets, which are the so-called hard parts, as shown in Fig. 1. A group of cables are routed from the armor cuff to the elbow upper bracket. In the design presented, three cables are used for the shoulder joint, with two cables connecting the elbow upper and lower brackets, as shown in Fig. 2.

The armor of the exoskeleton is anchored on the trunk of the user. On the back of the armor, a casing holds all springs. In this spring casing, an array of pre-loaded springs is setup to provide the forces for driving the motion of the arm. The spring box has individual switches for each spring to adjust the pre-load force based on the condition of the patient's disability. Three via points are designed on the armor to position the cables. The spring forces from the spring array are transferred through the cables to the elbow and shoulder joint of the human arm.

At the elbow joint, two anchoring nodes are designed on the elbow lower bracket, as shown in Fig. 2. Two cables linked to two springs from the anchoring point through the two via points on the elbow upper bracket. The force in the cable hereby can provide balance torque for the flexion motion of the elbow. The three anchoring points on the upper bracket are the attaching nodes for the spring cables which balance the shoulder joint.

In order to simplify the prototyping of the exoskeleton, the mechanical structures of the exoskeleton are developed, as shown in Figs. 1(b) and 2(b). The armor part is a welded assembly tubes to minimize weight. The elbow brackets made of aluminium are formed as assembly parts and cushioned on the inside to provide comfortable skin-contact. The purpose of the prototype is proof-of-concept and it will be further matured for actual patient use. 


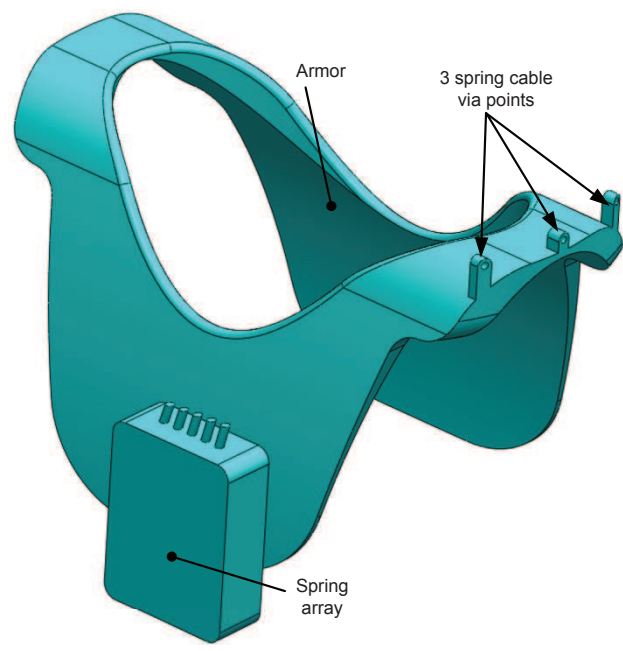

(a)

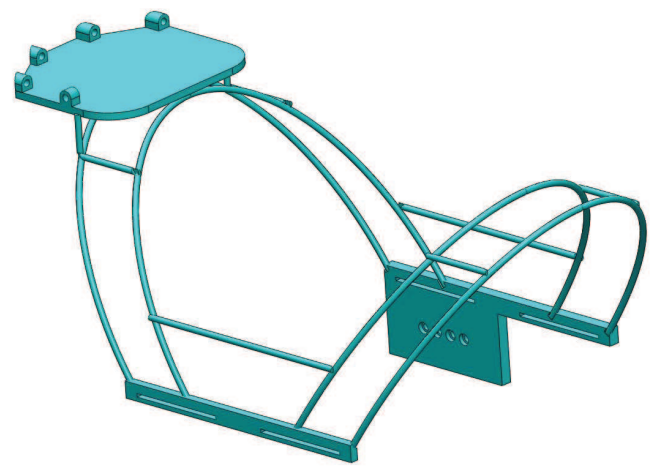

(b)

Figure 1: The armor part of the exoskeleton, (a) the profile of the armor part, (b) mechanical structure

\section{Modeling of the Human-exoskeleton System}

A human-exoskeleton is a type of biomechanical system where the exoskeleton works cooperatively with human muscles and nervous system. The interaction between the exoskeleton and the human body determines whether an exoskeleton can implement the desired functions. A central issue in the modeling work is thus to simulate the response of the human body subject to external forces/torques exerted by the exoskeleton.

The human-exoskeleton model is constructed by combination of the human arm and the exoskeleton model, schematically shown in Fig. 3. The figure only shows the kinematic structure of the humanexoskeleton model. The blue lines stand for the skeleton of a human arm, while the red ones represent the

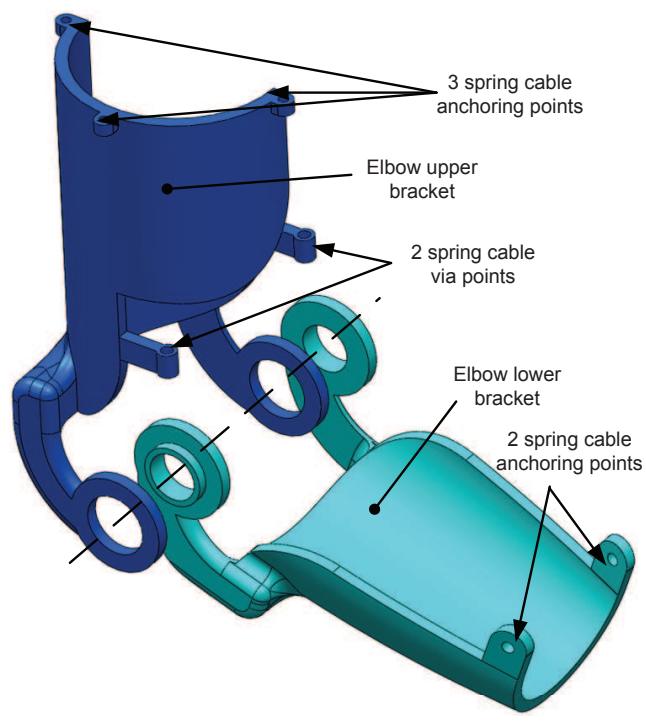

(a)

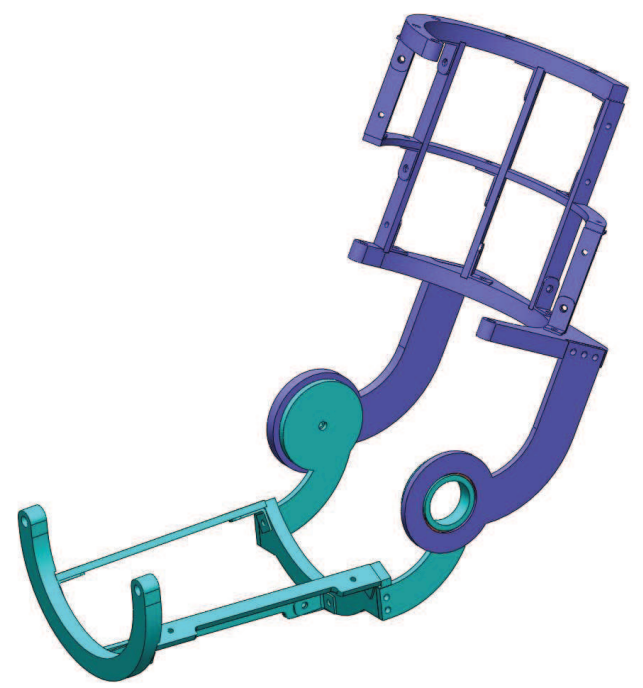

(b)

Figure 2: CAD embodiment of the exoskeleton, (a) the profile of the elbow bracket assembly, (b) mechanical structure.

exoskeleton. In the human arm model, only the glenohumeral joint (represented by three revolute joints $\theta_{1}$, $\left.\theta_{2}, \theta_{3}\right)$ and the elbow flexion joint $\theta_{4}$ are supported by the exoskeleton. The upper elbow bracket is connected to the upper arm through a translation-spherical joint, which allows the bracket to translate freely along the axis of the upper arm. The translational-spherical joint is represented as a spherical joint with three angles $\theta_{e 1}, \theta_{e 2}, \theta_{e 3}$, and one translational joint $p_{e 4}$. The upper and lower elbow brackets are connected to each other through a revolute joint $\theta_{e 5}$. The attachment of the lower bracket to the forearm is modeled as a 
revolute joint $\theta_{e 6}$. This kind of attachment allows the lower bracket to rotate around the pronation axis of the forearm.

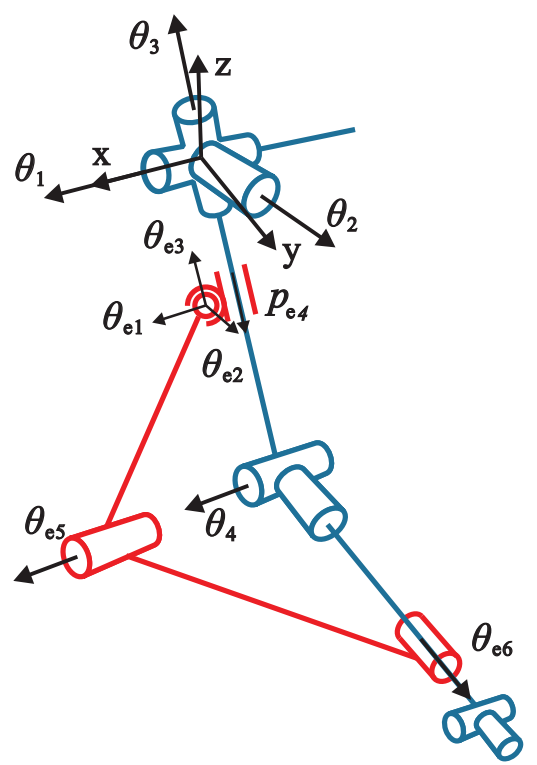

Figure 3: Coordinates and kinematic configuration of the human-exoskeleton system.

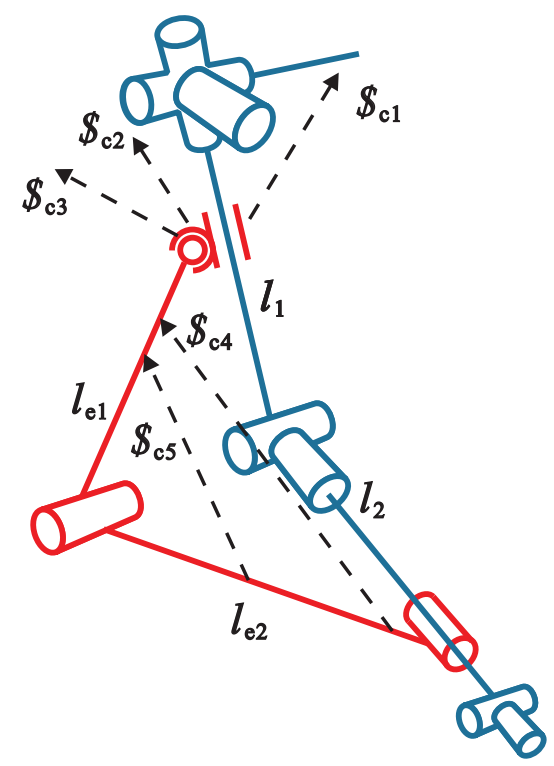

Figure 4: Cables used in the human-exoskeleton system.

The elbow bracket assembly is connected to an armor cuff, which is fixed to the trunk of the human model. The routing of the five cables is shown in Fig. 4 as dashed lines, while the arrows show the direction of cable tension. Three cables $\boldsymbol{\$}_{c 1}, \boldsymbol{\$}_{c 2}$, and $\boldsymbol{\$}_{c 3}$ are anchored from the upper elbow bracket and directed to the armor cuff. These three cables wrap over the glenohumeral joint. With given tensions they can provide supporting torque to the human shoulder. Two cables $\$_{c 4}$ and $\$_{c 5}$ connect the upper and lower elbow brackets.

\subsection{Human Arm Biomechanical Model}

In a musculoskeletal model, the human body is modeled as a multibody system, in which bones and joints are treated as mechanical links and joints, while muscles exert force on the system. It is known that the system is statically indeterminate, because the body contains more muscles than degrees-of-freedom. To resolve this problem, AMS utilizes optimization to compute the muscle and joint forces:

$$
\begin{array}{cl}
\min & G\left(\mathbf{f}^{(M)}\right) \\
\text { s.t. } & \mathbf{C f}=\mathbf{d}^{*} \\
& f_{i}^{(M)} \geq 0, \quad i \in\left\{1, \ldots, n^{(M)}\right\}
\end{array}
$$

where $\mathbf{f}=\left[\mathbf{f}^{(R)}, \mathbf{f}^{(M)}\right]$ is an $n$-dimensional vector of joint reaction forces $\mathbf{f}^{(R)}$ and muscle forces $\mathbf{f}^{(M)}$. The vector $\mathbf{d}^{*}$ is the external force including gravity and dynamic forces, and $\mathbf{C}$ is a coefficient matrix generated from the arm anatomy and muscle attachments. The objective function $G\left(\mathbf{f}^{(M)}\right)$ expresses the muscle recruitment criterion. The possible criteria include soft saturation, $\min / \max$ and polynomial muscle recruitment (Rasmussen et al., 2001). The polynomial criterion is adopted as

$$
G\left(\mathbf{f}^{(M)}\right)=\sum_{i}\left(\frac{f_{i}^{(M)}}{N_{i}}\right)^{p}
$$

where $N_{i}$ are normalization factors or functions, which take the form of muscle strength in this work. The power $p$ controls the synergy of muscles and $p=3$ is chosen in this case as it yields good results for most submaximal muscle efforts. The ratio $f_{i}^{(M)} / N_{i}$ refers to the muscle activity.

\subsection{Paralyzed Muscle}

To simulate paralyzed muscles in specific patient cases, certain muscles in the system must be disabled. The equilibrium equation in Eq. (1) can be rewritten as

$$
d_{j}=c_{j 1} f_{1}+\cdots+c_{j i} f_{i}+\cdots+c_{j n} f_{n}, j=1 \ldots m
$$

where $d_{j}$ denotes the $j$ th term of the vector $\mathbf{d}^{*}, c_{j i}$ is the term in the coefficient matrix $\mathbf{C}$, and $f_{i}$ is the $i$ th muscle force. 
If a muscle is disabled, its force is set to zero and the other muscle forces in Eq. (3) must increase to maintain equilibrium.

\subsection{Human-exoskeleton Dynamic Model}

The human arm skeleton together with the exoskeleton form a closed-loop chain system. The dynamic equation of motion of each segment is set up with the Newton-Euler equations

$$
\left[\begin{array}{cc}
m_{i} \mathbf{I} & \mathbf{0} \\
\mathbf{0} & \mathbf{J}_{i}^{\prime}
\end{array}\right] \ddot{\mathbf{q}}_{i}+\left[\begin{array}{c}
\mathbf{0} \\
\tilde{\omega}_{i}^{\prime} \mathbf{J}_{i}^{\prime} \omega_{i}^{\prime}
\end{array}\right]=\mathbf{g}_{i}
$$

where $\mathbf{q}_{i}$ is the vector of assembled coordinates for all segments, $\omega_{i}^{\prime}$ is the angular velocity measured in the body-fixed reference frame. The segment mass is denoted as $m_{i}$, and $\mathbf{J}_{i}^{\prime}$ is the inertia tensor with respect to the centroidal body-frame. The right-hand side, $\mathbf{g}_{i}$, contains muscle forces, joint reaction forces, and known applied forces $\mathbf{g}_{i}^{(\mathrm{app})}$. For the system with human arm and the exoskeleton, the external force $\mathbf{d}^{*}$ in Eq. (1) can be expressed as

$$
\mathbf{d}_{i}^{*}=\mathbf{g}_{i}^{(\mathrm{app})}-\left[\begin{array}{cc}
m_{i} \mathbf{I} & \mathbf{0} \\
\mathbf{0} & \mathbf{J}_{i}^{\prime}
\end{array}\right] \ddot{\mathbf{q}}_{i}-\left[\begin{array}{c}
\mathbf{0} \\
\tilde{\omega}_{i}^{\prime} \mathbf{J}_{i}^{\prime} \omega_{i}^{\prime}
\end{array}\right]
$$

The detailed explanation can be seen in (Damsgaard et al., 2006).

Taking into consideration of spring forces, the righthand side of the dynamic equation in Eq. (1) is then calculated by

$$
\mathbf{d}_{i}=\mathbf{d}_{i}^{*}+\mathbf{E}(\mathbf{q})^{\mathrm{T}} \mathbf{t}_{c}
$$

where vector $\mathbf{t}_{c}$ contains the tensions of the incorporated springs. The coefficient matrix $\mathbf{E}$ is generated from the installation of springs in the exoskeleton and the exoskeleton attachment to the arm.

The muscle recruitment in Eq. (1) then becomes

$$
\begin{array}{cl}
\min & G\left(\mathbf{f}^{(M)}\right) \\
\text { s.t. } & \mathbf{C f}=\mathbf{d} \\
& f_{i}^{(M)} \geq 0, \quad i \in\left\{1, \ldots, n^{(M)}\right\}
\end{array}
$$

\subsection{Spring Tension}

As shown in Fig. 5, the green lines represent the cables for transferring forces. In this design, two springs are used to drive the elbow joint and three springs to drive the shoulder joint. The cable tension generated by the spring is thus calculated by

$$
\mathbf{t}_{c}=\mathbf{t}_{\text {pre }}+\mathbf{k} \Delta \mathbf{l}
$$

with

$$
\mathbf{k}=\left[\begin{array}{ccccc}
k_{g h 1} & & & & 0 \\
& k_{g h 2} & & & \\
& & k_{g h 3} & & \\
0 & & & k_{e l 1} & \\
0 & & & & k_{e l 2}
\end{array}\right]
$$

where $\mathbf{t}_{\text {pre }}$ denotes pre-load of the spring, $\mathbf{k}$ is the spring stiffness matrix. $\Delta \mathrm{l}$ is the vector of the spring elongations.

The pre-load tension of the spring is

$$
\mathbf{t}_{\text {pre }}=\mathbf{k} \mathbf{l}_{\text {pre }}
$$

with $\mathbf{l}_{\text {pre }}$ be the pre-load length of the spring.

Combining Eqs. (8) and (10), the cable tension can be calculated by

$$
\mathbf{t}_{c}=\mathbf{k}\left(\mathbf{l}_{\text {pre }}+\Delta \mathbf{l}\right)
$$

Here, we divide the elongation of the spring into two parts. The reason is that at any initial state of the exoskeleton, some cable(s) should be taut to compensate the weight of the arm. The variation of the spring length $\Delta \mathbf{l}$ only changes according to the positions of the attaching points. The pre-load length $\mathbf{l}_{\text {pre }}$ provides us a way to manipulate the pre-load tension with respect to different load cases.

\section{Implementation of Human-exoskeleton Model}

The motion of the musculoskeletal model is based on motion capture data. Brachial plexus injury, arising typically from falls and traffic accidents, is selected as the patient case of the exoskeleton (Glanze et al., 1990).

\subsection{Implementation in AnyBody}

The dynamic equations of the biomechanical system are nonlinear and complex. Hence, the AnyBody Modeling System is employed to execute dynamics analysis. Besides, the reactions of the human body to the exoskeleton are the core design criteria, the modeling of which is the major feature of AnyBody. The biomechanical model built in AnyBody is shown in Fig. 5. The exoskeleton model is built in SolidWorks and then exported to AnyBody. Several reference nodes are defined on the human model for placing the armor cuff and brackets of the exoskeleton. The mass properties of the exoskeleton can be calculated in SolidWorks and exported to AnyBody afterwards. The interaction joints between the arm and exoskeleton are defined according to the biomechanical kinematic model. 


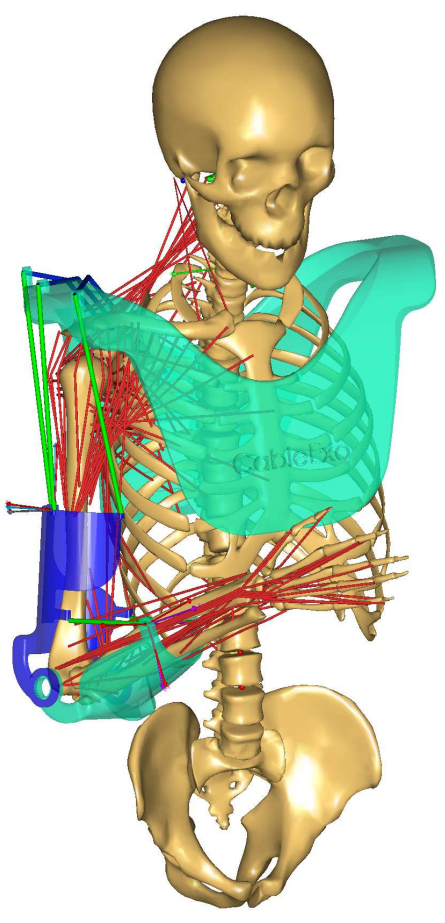

Figure 5: A wearable spring-loaded cable-driven exoskeleton with a musculoskeletal model.

The musculoskeletal right arm model is derived from the repository models (Version 1.4) in AnyBody. The whole musculoskeletal model is comprised of 39 joints and 134 muscles.

Several special configurations are made to the human-exoskeleton model in AnyBody:

1. The exoskeleton does not support the wrist joint and the pronation of the forearm, even though these are affected by the paralysis depending on the location of the lesion. To focus the design on the supported DoFs, the biomechanical model supports the wrist flexion, wrist abduction and the elbow pronation artificially by reaction forces. This corresponds to supporting the wrist joint and elbow pronation with orthotics.

2. In the modeling, the forearm is allowed to pronate/supinate inside the elbow lower bracket to mimic the significant motion of the skin above the bones. Support of the forearm pronation/supination would require development or an interface between the exoskeleton and the forearm capable of supporting torsion.

3. The human arm model is used to simulate particular lesion cases. Each piece of muscle in the arm is linked to a corresponding nerve as explained in the next section. The muscle can be enabled/disabled depending on the lesion type.

\subsection{Design Case}

The brachial plexus is a network of nerves that transfers signals from the spinal cord to the shoulder and upper limb. These nerves root from the fifth to the eighth cervical (C5-C8), and first thoracic (T1) spinal nerves, as illustrated in Fig. 6(a). They innervate the muscles and skin of the chest, shoulder and upper limb. Injuries of brachial plexus, or lesions, often caused by trauma conditions such as traffic accidents can have serious effect on the mobility of limbs (Shveiky et al., 2010).

The branches of the brachial plexus and their associated muscles are listed in Table 1, sorted with respect to their roots. The spinal nerves and their cord related to brachial plexus are shown in Fig. 6(a) and Fig. 6(b).

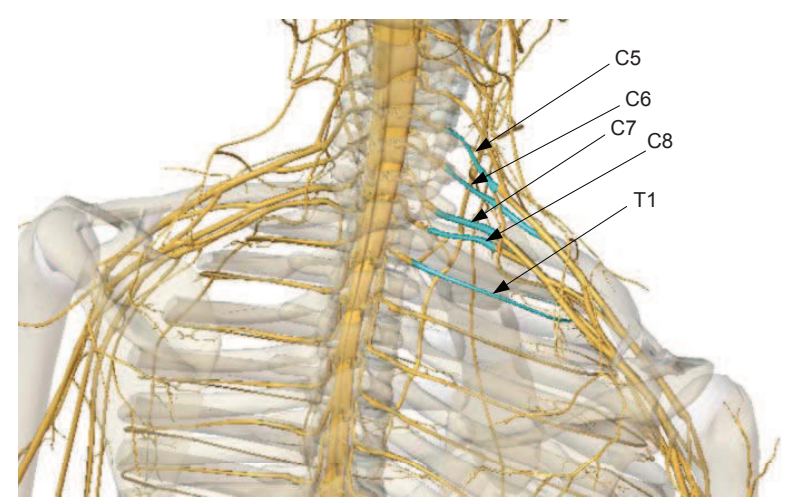

(a)

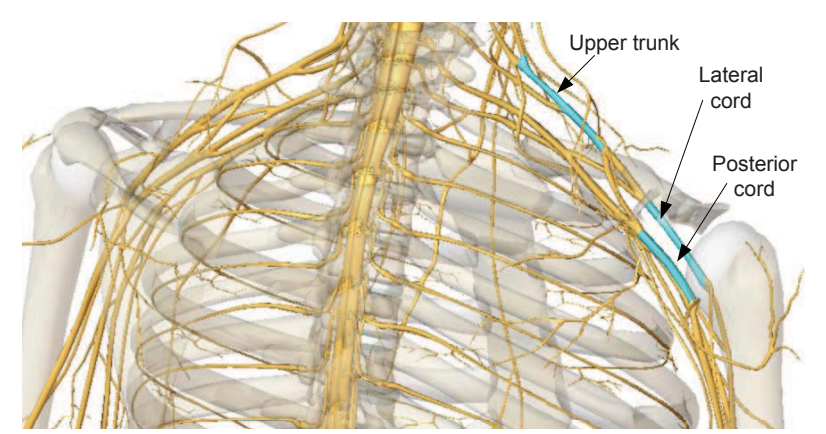

(b)

Figure 6: The spinal nerve cords of the nervous system.

\subsection{Motion of a Human Arm}

In order to actuate the musculoskeletal human arm model, realistic motion data is needed. In this work, a customized motion capture system is built by two Kinect $^{T M}$ sensors. A motion of picking up a cup and drinking is captured within 3 seconds, as shown in Fig. 7. The payload is $0.5 \mathrm{~kg}$ carried by the hand.

The joint movements realizing the hand trajectory 
Table 1: Brachial plexus branches associated to different arm muscles (Moore and Agur, 2007).

\begin{tabular}{|c|c|c|}
\hline No. & Nerve & Muscles \\
\hline 1 & C5 & $\begin{array}{l}\text { deltoid, teres minor, triceps brachii, supinator, anconeus, the extensor muscles } \\
\text { of the forearm, brachioradialis, subscapularis, teres major, pectoralis major, } \\
\text { coracobrachialis, brachialis, biceps brachii, serratus anterior, rhomboid muscles, } \\
\text { levator scapulae }\end{array}$ \\
\hline 2 & C6 & $\begin{array}{l}\text { deltoid, teres minor, triceps brachii, supinator, anconeus, the extensor muscles } \\
\text { of the forearm, brachioradialis, subscapularis, teres major, pectoralis major, } \\
\text { coracobrachialis, brachialis, biceps brachii, serratus anterior, latissimus dorsi }\end{array}$ \\
\hline 3 & $\mathrm{C} 7$ & $\begin{array}{l}\text { triceps brachii, supinator, anconeus, the extensor muscles of the forearm, bra- } \\
\text { chioradialis, latissimus dorsi, pectoralis major, coracobrachialis, brachialis, bi- } \\
\text { ceps brachii, serratus anterior }\end{array}$ \\
\hline 4 & $\mathrm{C} 8$ & $\begin{array}{l}\text { triceps brachii, supinator, anconeus, the extensor muscles of the forearm, bra- } \\
\text { chioradialis, latissimus dorsi }\end{array}$ \\
\hline 5 & $\mathrm{~T} 1$ & $\begin{array}{l}\text { triceps brachii, supinator, anconeus, the extensor muscles of the forearm, bra- } \\
\text { chioradialis }\end{array}$ \\
\hline 6 & $\begin{array}{l}\text { posterior } \\
\text { cord }\end{array}$ & $\begin{array}{l}\text { deltoid, teres minor, triceps brachii, supinator, anconeus, the extensor muscles } \\
\text { of the forearm, brachioradialis, subscapularis, teres major, latissimus dorsi }\end{array}$ \\
\hline 7 & $\begin{array}{l}\text { lateral } \\
\text { cord }\end{array}$ & pectoralis major, coracobrachialis, brachialis, biceps brachii \\
\hline 8 & $\begin{array}{l}\text { upper } \\
\text { trunk }\end{array}$ & supraspinatus, infraspinatus \\
\hline 9 & root & serratus anterior, rhomboid muscles, levator scapulae \\
\hline
\end{tabular}

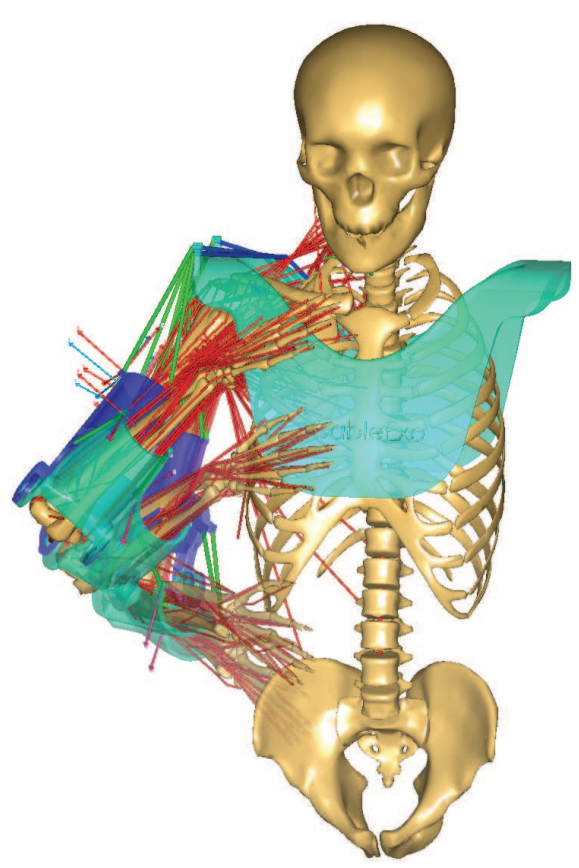

Figure 7: The motion of picking up a cup and drinking in 3 seconds. are depicted in Fig. 8. As the exoskeleton does not support the motion of the elbow pronation and the wrist joint, only the other four joints motion of the human arm are captured and utilized in the simulation.

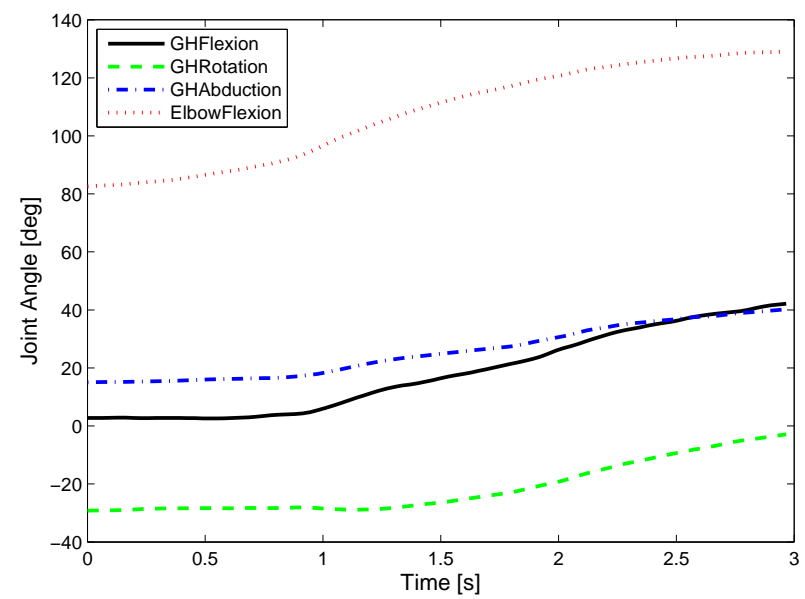

Figure 8: Joint angles in the motion of lifting a cup. (GH denotes the glenohumeral joint.)

A simulated MMACT over the motion for an ablebodied individual is displayed in Fig. 9 


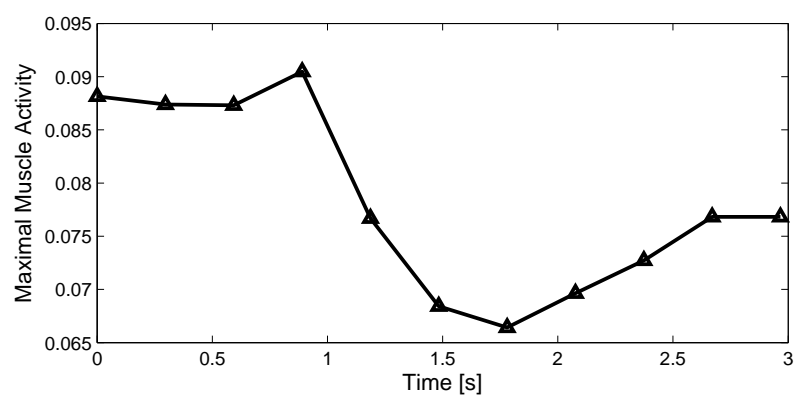

Figure 9: Muscle activity in the motion of picking up a cup.

\subsection{Maximal Muscle Activation}

The simulation of the brachial plexus injury is categorized according to its root and origin. Muscle activity is defined as the ratio between the instantaneous muscle force and the instantaneous muscle strength. When the muscle activity of any muscle exceeds 1 , that muscle has insufficient strength to complete the required motion. To allow the simulation to complete in cases of inadequate muscle strength, weak artificial muscles have been added to the joints, thus allowing all the cases of lesions to be simulated, albeit in some cases with very high activation levels.

The maximal muscle activation (MMACT) is calculated for different nerve lesion conditions. The arm muscles are categorized into groups, as shown in Table 1. For example, if the nerve root $\mathrm{C} 7$ has a lesion, all the muscles related to $\mathrm{C} 7$ will be paralyzed. The calculated maximal MMACTs of the different nerve lesions are shown in Fig. 10. Note that the case BASE refers to the motion without any nerve lesion.

It is found that paralyzing nerve C5 or C6 will lead to very high MMACT. This is reasonable as these lesions paralyze most of the functional muscles in the upper arm and shoulder. The high required muscle activity indicates that if there is lesion at C5 or C6, it might be hard to restore motion with a purely passive exoskeleton. The maximal MMACTs of nerve lesions C8, T1, Posterior cord, Lateral cord, Upper trunk and Root do not exceed 1 , such that these nerve lesions do not need the assistance of the exoskeleton. The case C7 with a maximal MMACT of 25.1 is selected for investigation as to be described in Sec. 6 .

\section{Design Optimization}

With the model of the human-exoskeleton system, we are able to simulate and analyze the muscle activity for given motion and design parameters. As the springcable-driven exoskeleton utilizes five springs in the pro-

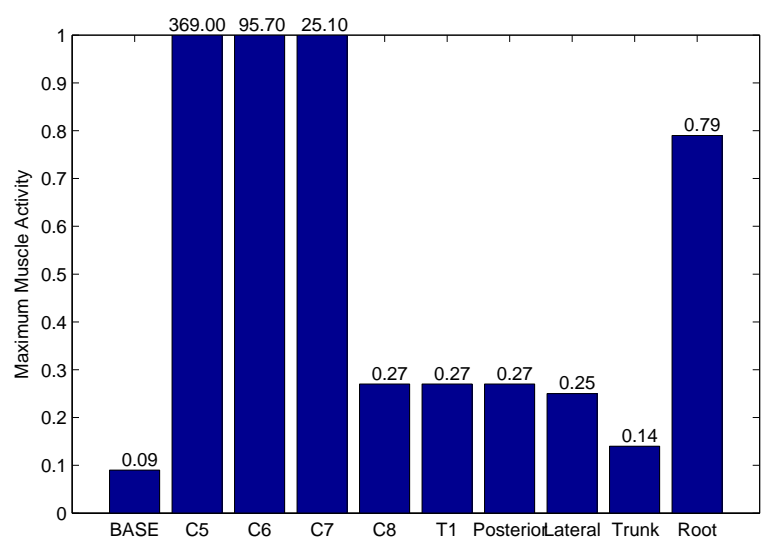

Figure 10: Maximal MMACT of different nerve lesion cases.

posed design, for which the stiffness has to be selected to provide assistive function, we further developed an optimization method to select proper spring stiffness. In the optimization problem, the objective of the exoskeleton is to reduce the maximal MMACT over the entire motion. The objective function is defined as

$$
\begin{aligned}
& \min _{\mathbf{x}} f(\mathbf{x})=\max _{t}\left\{\max _{i}\left\{\frac{f_{i}^{(M)}}{N_{i}}\right\}\right\} \\
& \mathbf{x}=\left[k_{g h 1}, k_{g h 2}, k_{g h 3}, k_{e l 1}, k_{e l 2}\right] \\
& \text { s.t. } \min \quad G\left(\mathbf{f}^{(M)}\right) \\
& \mathbf{C f}=\mathbf{d} \\
& f_{i}^{(M)} \geq 0, \quad i \in\left\{1, \ldots, n^{(M)}\right\}
\end{aligned}
$$

where the design variables $\mathbf{x}$ refer to the five springs linked to the cables $\boldsymbol{\$}_{c 1}, \boldsymbol{\$}_{c 2}, \boldsymbol{\$}_{c 3}, \boldsymbol{\$}_{c 4}$ and $\boldsymbol{\$}_{c 5}$ in Fig. 4. The optimization problem is solved by the Complex method (Box, 1965; Guin, 1968) with its implementation in AnyBody. This optimization problem is wrapped around the full inverse dynamic analysis of the model. In each iteration, the maximal MMACT is calculated over the motion duration $t$ after inverse dynamics is completed.

The overall structure of simulation and design optimization is illustrated in Fig. 11.

\section{Simulation and Optimization Results}

\subsection{Optimization Results}

For the case $\mathrm{C} 7$ in the motion of picking up a cup and drinking, we set a population number of 30 to the 


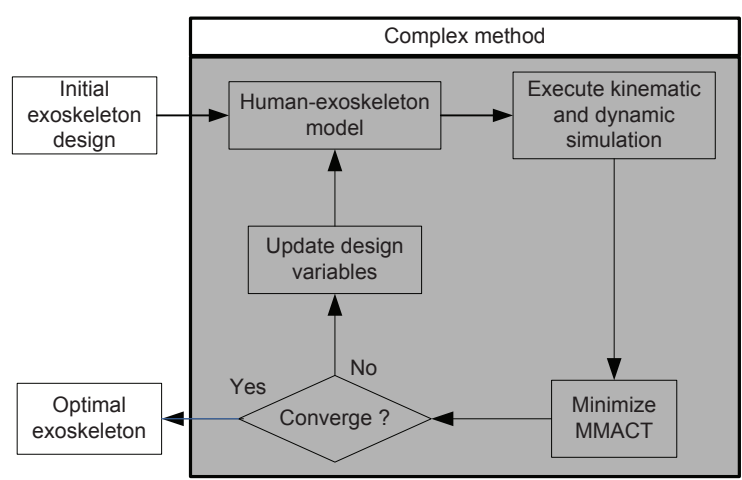

Figure 11: Routine of design optimization.

Complex method. The objective convergence tolerance is 0.001 , and the convergence tolerance for the design variables is 0.1 . The optimization reduces the maximal MMACT from 25.1 of the case without assistance to 0.58 with the exoskeleton. The convergence of the maximal MMACT is plotted in Fig. 12.

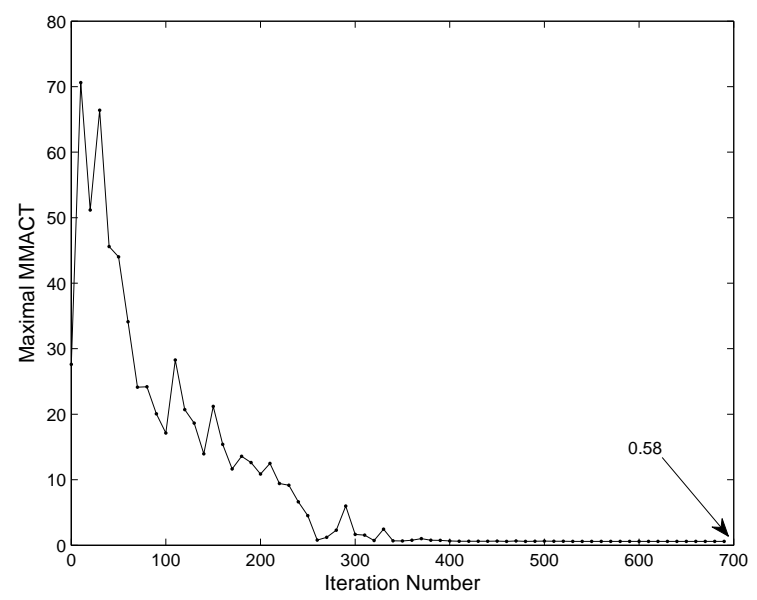

Figure 12: Optimization of the maximal MMACT.

In the optimization case, all the pre-load lengths of the five springs are set to $l_{\text {pre }}=0.06 \mathrm{~m}$. The optimal design variables are obtained as

$$
\mathbf{x}=[1473.4,0.3,0.03,102.0,1979.0] \mathrm{N} / \mathrm{m}
$$

The iteration history of the stiffness coefficients of the five springs are plotted in Fig. 13, which can be used to select springs for the exoskeleton.

\subsection{Cable Tension}

The optimal spring stiffness $k_{g h 2}=0.3 \mathrm{~N} / \mathrm{m}$ and $k_{g h 3}=0.03 \mathrm{~N} / \mathrm{m}$ for cables 2 and 3 are negligible. The cable tensions for the other three springs during the motion of picking up a cup are plotted in Fig. 14.

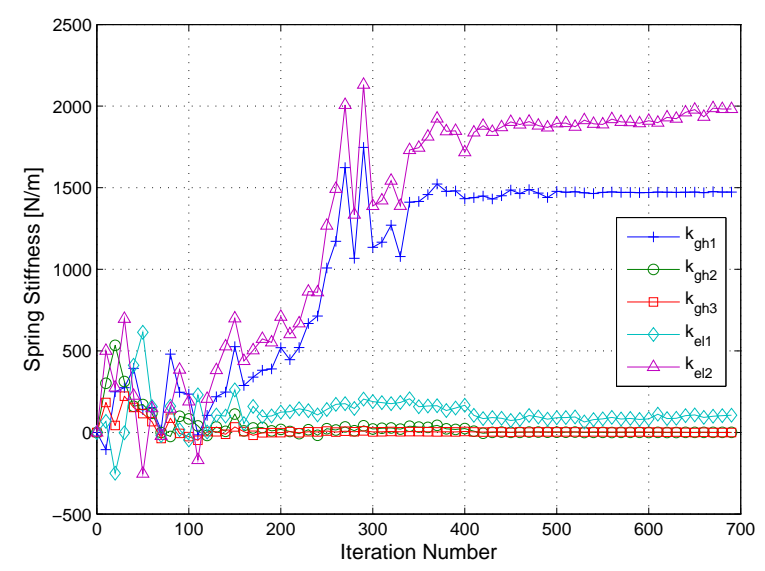

Figure 13: Convergence of the stiffness coefficients.

Cable 1 is the only one for supporting the glenohumeral joint. As cable 1 compensates almost the weight of the whole arm and the exoskeleton elbow brackets, it requires the highest tension. The tensions of cables 4 and 5 fall down to zero in the later stage of the motion as the two springs are returning to their slack states. Since the cable can only pull, the cable tensions will be always larger than zero.

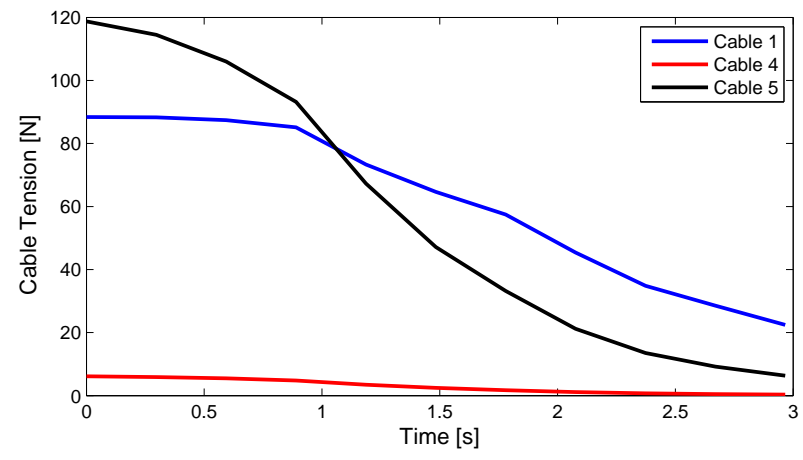

Figure 14: Cable tension on the optimal exoskeleton.

\subsection{Arm Reactions}

With the optimal parameters, the MMACT is calculated for the case without exoskeleton and the one with the optimal design, as plotted in Fig. 15. Wearing the optimal designed exoskeleton, a big reduction of the MMACT can be seen in the human body. After optimization, the MMACT of the arm is always below 1 in the motion. The patient with a $\mathrm{C} 7$ nerve lesion can wear the exoskeleton and therefore perform the activity of lifting a cup and drinking. 


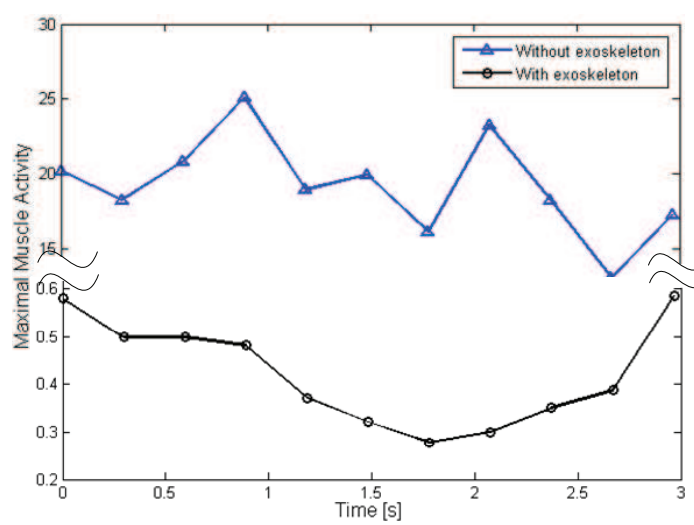

Figure 15: The comparison of the MMACT after optimization.

\section{Discussions}

The result of the combined simulation and optimization process was very satisfactory in the sense that the simulated model is enabled by the exoskeleton to perform the desired task. However, the analysis is based on an ideal situation in which the nerve lesion and its precise effect on the muscles are known. Actual patient cases may be more complex or less well-defined. Also, the model does not take dissipative effects such as friction in the cables and joints, visco-elastic effects in the interfaces with soft tissue or sensitivity of the solution to design tolerances into account. It is therefore likely that significant practical experimentation and design maturation are necessary before exoskeletons of this type reach their full potential for specific patients with specific lesions.

The passive type of exoskeleton has the distinct advantage of simplicity over its active counterparts, and the complex control, safety and power supply issues following from the use of electrical motors and controllers are eliminated. Due to the absence of rigid links around the shoulder joint, the exoskeleton does not constrain the free motion of the shoulder.

The optimization results show that only three of the springs are exploited for this patient and movement case. However, it does not mean that the other two springs are redundant; they become useful for other movements. The selected arm motion for the design optimization is quasi-static, and dynamic effects may simultaneously limit and extend the usability of the exoskeleton. Users of advanced orthotics are often able to extend their control beyond the available support by using dynamic effects to their advantage. On the other hand, the simulation provides no guarantee that the proposed exoskeleton is applicable to fast arm motion.

\section{Conclusions}

An approach to design exoskeletons through biomechanics simulation was proposed. It involves an integrated human-exoskeleton model to simulate and optimize the wearable exoskeleton. The model can simulate the biomechanics of the human arm in the presence of partial paralysis. The integration of the human and exoskeleton models reveals the interaction between two individual models, and help to optimize the exoskeleton with respect to the performance of the human model. The specialized model can be used to design different types of exoskeletons based on different neuromuscular injuries. A spring-loaded and cable-driven exoskeleton was designed by the developed approach.

The approach provides a convenient and efficient way to design and develop wearable exoskeletons. Through biomechanics simulations, many parameters of exoskeletons can be obtained and evaluated based on the reactions of the human body model. Nevertheless, design simulation alone is insufficient so a prototype will be built and experiments with real patients' activities of daily living will be conducted. The results of these efforts will likely lead to modifications towards the final design.

\section{Acknowledgements}

The project belongs to a strategic platform for research and innovation, namely, Patient @ home, which is funded by The Danish Agency for Science, Technology and Innovation.

\section{References}

Agrawal, S. K., Dubey, V. N., Gangloff, J. J., Brackbill, Y., Mao, Y., and Sangwan, V. Design and optimization of a cable driven upper arm exoskeleton. Journal of Medical Devices, 2009. 3:031004-1-031004-8. doi:10.1115/1.3191724.

Bai, S. and Rasmussen, J. Modelling of physical human-robot interaction for exoskeleton designs. In J. C. Samin and P. Fisette, editors, Proc. of Multibody Dynamics 2011, ECCOMAS Thematic Conference. Brussels, Belgium, pages 1-7, 2011.

Box, M. J. A new method of constrained optimization and a comparison with other methods. Computer Journal, 1965. 8:42-52. doi:10.1093/comjnl/8.1.42.

Carignan, C., Tang, J., and Roderick, S. Development of an exoskeleton haptic interface for virtual task 
training. In Proc. of IEEE/RSJ Inter. Conf. on Intelligent Robots and Systems. pages 3697-3702, 2009. doi:10.1109/IROS.2009.5354834.

Damsgaard, M., Rasmussen, J., Christensen, S. T., Surma, E., and Zee, M. d. Analysis of musculoskeletal systems in the Anybody Modeling System. Simulation Modelling Practice and Theory, 2006. 14:1100-1111. doi:10.1016/j.simpat.2006.09.001.

Gallagher, W., Ding, M., and Ueda, J. Relaxed individual control of skeletal muscle forces via physical human-robot interaction. Multibody System Dynamics, 2013. 30:77-99. doi:10.1007/s11044-013-9362-y.

Garrec, P., Friconneau, J. P., Measson, Y., and Perrot, Y. ABLE, an innovative transparent exoskeleton for the upper-limb. In Proc. of IEEE/RSJ Inter. Conf. on Intelligent Robots and Systems. pages 1483-1488, 2008. doi:10.1109/IROS.2008.4651012.

Glanze, W. D., Anderson, K. N., and Anderson, L. E. Mosby's Medical, Nursing, and Allied Health Dictionary (3rd ed.). The C.V. Mosby Co., St. Louis, Missouri, USA, 1990.

Guin, J. A. Modification of the complex method of constrained optimization. Computer Journal, 1968. 10:416-417. doi:10.1093/comjnl/10.4.416.

Haumont, T., Rahman, T., Sample, W., King, M. M., Church, C., Henley, J., and Jayakumar, S. Wilmington robotic exoskeleton: a novel device to maintain arm improvement in muscular disease. Journal of Pediatric Orthopedics, 2011. 31(5):e44-e49. doi:10.1097/BPO.0b013e31821f50b5.

Lee, L. F., Narayanan, M. S., Kannan, S., Mendel, F., and Krovi, V. N. Case studies of musculoskeletalsimulation-based rehabilitation program evaluation. IEEE Transactions on Robotics, 2009. 25(3):634638. doi:10.1109/TRO.2009.2019780.

Lenzi, T., Vitiello, N., Rossi, S. M. M. D., Roccella, S., Vecchi, F., and Carrozza, M. C. NEUROExos: a variable impedance powered elbow exoskeleton. In Proc. of IEEE Inter. Conf. on Robotics and Automation. Shanghai, China, pages 1419-1426, 2011. doi:10.1109/ICRA.2011.5979866.

Lo, H. S. and Xie, S. Q. Exoskeleton robots for upper-limb rehabilitation: State of the art and future prospects. Medical Engineering \& $\quad$ Physics, 2012. 34(3):261-268. doi:10.1016/j.medengphy.2011.10.004.

Mao, Y. and Agrawal, S. K. Design of a cable-driven arm exoskeleton (CAREX) for neural rehabilitation.
IEEE Transactions on Robotics, 2012. 28(4):922931. doi:10.1109/TRO.2012.2189496.

Moore, K. L. and Agur, A. M. Essential Clinical Anatomy (3rd ed.). Lippincott Williams \& Wilkins, Baltimore, USA, 2007.

Nef, T., Guidali, M., and Riener, R. ARMinIII - arm therapy exoskeleton with an ergonomic shoulder actuation. Applied Bionics and Biomechanics, 2009. 6(2):127-142. doi:10.1080/11762320902840179.

Perry, J. C., Rosen, J., and Burns, S. Upperlimb powered exoskeleton design. IEEE/ASME Transactions on Mechatronics, 2007. 12(4):408-417. doi:10.1109/TMECH.2007.901934.

Rahman, T. and et al. Passive exoskeletons for assisting limb movement. Journal of Rehabilitation Research 83 Development, 2006. 43(5):583-590. doi:10.1682/JRRD.2005.04.0070.

Rasmussen, J., Damsgaard, M., and Voigt, M. Muscle recruitment by the min/max criterion: A comparative numerical study. Journal of Biomechanics, 2001. 34(3):409-415. doi:10.1016/S0021-9290(00)00191-3.

Ren, Y., Park, H. S., and Zhang, L. Q. Developing a whole-arm exoskeleton robot with hand opening and closing mechanism for upper limb stroke rehabilitation. In Proc. of IEEE Inter. Conf. on Rehabilitation Robotics. pages 761-765, 2009. doi:10.1109/ICORR.2009.5209482.

Shveiky, D., Aseff, J. N., and Iglesia, C. B. Brachial plexus injury after laparoscopic and robotic surgery. The Journal of Minimally Invasive Gynecology, 2010. 17(4):414-420. doi:10.1016/j.jmig.2010.02.010.

Vertechy, R., Frisoli, A., Dettori, A., Solazzi, M., and Bergamasco, M. Development of a new exoskeleton for upper limb rehabilitation. In Proc. of IEEE/RSJ Inter. Conf. on Intelligent Robots and Systems. pages 188-193, 2009. doi:10.1109/ICORR.2009.5209502.

Wolbrecht, E. T., Chan, V., Reinkensmeyer, D. J., and Bobrow, J. E. Optimizing compliant, modelbased robotic assistance to promote neurorehabilitation. IEEE Transactions on Neural Systems and Rehabilitation Engineering, 2008. 16(3):286-297. doi:10.1109/TNSRE.2008.918389.

Wu, T. M., Wang, S. Y., and Chen, D. Z. Design of an exoskeleton for strengthening the upper limb muscle for overextension injury prevention. Mechanism and Machine Theory, 2011. 46(12):1825-1839. doi:10.1016/j.mechmachtheory.2011.08.003. 